\title{
Coating of Single Cells towards Biomedical Applications
}

\author{
Sung Ho Yang* \\ Associate professor, Korea National University of Education, Korea
}

Received: March 19, 2018; Published: April 05, 2018

*Corresponding author: Sung Ho Yang, Associate professor, Korea National University of Education, Korea, Email: sunghoyang@knue.ac.kr

\section{Introduction}

In 2002, a pioneering work on single cell coating with macromolecules through layer-by-layer (LbL) method was published in Langmuir [1]. Yeast cells were alternately coated with cationic and anionic macromolecules. Even after coating, the cells still survived. Inspired by the work, multilayered macromolecules have been used for coating various types of microbial cells and endospores [2]. Although the survival of microbial cells in the artificial coat was interesting of itself, the impact of works was under evaluated in the biomedical fields because the method was limited to microbial cells and the macromolecular coating had no particular roles. The two limitations have been gradually solved by developing functional coating materials and applying the method to mammalian cells. The function of coating materials was mainly improved by shifting from organic macromolecules to inorganic materials, in other words, from soft to hard materials Tang et al. [3-6]. crystalized calcium phosphate on the multilayer-coated yeast cells, leading to rigid artificial shells [7]. Choi et al. synthesized silica on the multilayer-coated yeast cells in a biomimetic manner, which was the first report on coating living cells with covalent bonded inorganic materials [8-10]. And chrollera cells were coated with Titania using catalytic peptides [11].

The strong coating has provided native cells with superior properties which were beneficial for protecting the cells and changing inherent cellular behaviors. For instance, coated cells survived longer than native cells under unfavorable conditions and the artificial shell protected the cells from foreign aggressions. The cell division could be retarded presumably because a physical barrier caused by the shell suppressed the cell division and decrease permeability of nutrients across the coats. Although organic macromolecules are mostly weaker than inorganic materials, strong organic materials also have been developed to coat yeast cells. For example, polydopamine inspired by adhesive proteins in mussels and iron ion-tannic acid complex inspired by wine staining were successfully applicable to coating microbial cells $[12,13]$. The organic coats were capable of protecting cells and changing cellular behaviors.
On the other hand, cell-coating methods have been modified to be more biocompatible and applied to mammalian cells which were considered more difficult to manipulate with chemical treatments because the cells are more fragile than microbial cells. Despite the disadvantage, various types of LbL multilayers were used for coating mammalian cells such as red blood cells [14], fibroblasts [15], mesenchymal stem cells [16], breast cancer cells [17], and lymphocytes [18]. Chaikof et al. successfully coated pancreatic islets with poly-L-lysine linked to poly (ethylene glycol) $[19,20]$. The report showed us a strategy that toxic materials are also applicable to mammalian cells through the redaction of their toxicity by manipulating the materials biocompatibly. Akashi et al. coated human fibroblast cells, human umbilical vein endothelial cells, human hepatocellular carcinoma cells with multilayers composed of fibronectin (FN) and gelatin (G) through biospecific interactions [21]. By taking benefit of biocompatibility of biomaterials, the mammalian cells were coated without damage. The coated cells were packed into tissues with high density, which are applicable to tissue engineering. The coated cells were used for fabrication of artificial tissues with vascular networks, constructed by cocultivation of human umbilical vein-derived vascular endothelial cells between normal human dermal fibroblasts [22]. The artificial tissue was implanted into nude mice, resulting in connectivity between host and implanted tissue. Choi et al. expanded biomimetic silica synthesis to HeLa cells, which was useful for protection and control of the cells [23]. While native HeLa cells are grown by attaching onto the surface of cell culture flask, silica-coated cells were floated in culture media. The cell in an adhesive nature was changed to be a floating cell, which implies that the coating changed an inherent nature of the cells. Human Jurkat T cells were coated with titania while maintaining their viability and inherent functions [24]. The iron ion-tannic acid complex was also applied to coating mammalian cells [25].

There are useful biomedical applications for coating of virus although the virus is not a living thing. Virus is considered as a promising vector for gene therapy because of their inherent 
immunogenicity and strong transgene expression. However, it has some obstacles including preexisting antivector immunity and low efficiency of viral gene delivery. Tang et al. [26] coated Adenovirus serotype 5 with calcium phosphate and preexisting anti-Ad5 immunity was suppressed by the coating [26]. The other advantage of coated virus is increased thermal tolerance. The virus stabilized by silica coating was prolonged its infectivity by $\sim 10$ fold at room temperature while a similar result was obtained by storing native ones at $4^{\circ} \mathrm{C}$ [27]. Prolonged infectivity was mainly obtained by restricting molecular mobility of virion structure with silica nanoanchors. The enhanced thermal stability is practically important for long-term storage of virus vaccine. In commercial viewpoints, coated virus needs no refrigeration in its delivery, which is beneficial to save the cost in worldwide spread of vaccine. Human enterovirus 71 was also coated with alumina, which enhanced thermostability and immunogenicity [28].

Single cell coating is still approaching to more practical way towards biomedical applications. The coated cell has potential in the field of biomedical sciences. a)Protection of the cell would improve success rate of tissue implantation $[3,4,6]$. b)Enhanced viability gives a time enough to settle down implanted cells in a tissue of host body. The coating can also reduce immune-rejection by blocking direct contact between implanted cells and host tissue. c)Control of cell-cycle is beneficial to build up cell-based sensors which are integrated with electronic circuits and cells $[9,12]$. Durability of the sensors is mostly depended on viability of the cell and cell-cycles because cell death or cell growth will cause malfunction and bust the device. d)Coated virus could be used for a carrier for gene therapy because of high infectivity and low immune-rejection [26]. And stabilized virus is practically useful for long-term storage of vaccines because of its high thermal tolerance $[27,28]$. e)Coated cells have great potential in fundamental biological researches. For instance, the cells could be used for studying single cell behavior and cell-to-cell communication because the cells are isolated from outer environments including other cells $[3,6]$. Although there are still challenging issues on use of coated cells for biomedical applications, the strategies are evolving to more practical and biocompatible ways.

\section{References}

1. Diaspro A, Silvano D, Krol S, Cavalleri O, Gliozzi A (2002) Single living cell encapsulation in nano-organized polyelectrolyte shells. Langmuir 18(13): 5047-5050.

2. Fakhrullin RF, Zamaleeva AI, Minullina RT, Konnova SA, Paunov VN (2012) Cyborg cells: functionalization of living cells with polymers and nanomaterials. Chemical Society Reviews 41: 4189-4206.

3. Yang SH, Hong D, Lee J, Ko EH, Choi IS (2013) Artificial spores: Cytocompatible encapsulation of individual living-cells within thin, tough artificial-shells. Small 9(2): 178-186.

4. Park JH, Yang SH, Lee J, Ko EH, Hong D, et al. (2014) Nanocoating of single cells: from maintenance of cell viability to manipulation of cellular activities. Advanced Materials 26(13): 2001-2010.

5. Cho WK, Yang SH (2015) Bio-inspired formation of silica thin films: from solid substrates to cellular interfaces. European Journal of Inorganic Chemistry 2015: 4481-4494.
6. Park JH, Hong D, Lee J, Choi IS (2016) Cell-in-shell hybrids: chemical nanoencapsulation of individual cells. Accounts of Chemical Research 49(5): 792-800.

7. Wang B, Liu P, Jiang W, Pan H, Xu X, Tang R (2008) Yeast cells with an artificial mineral shell: protection and modification of living cells by biomimetic mineralization. Angewandte Chemie International Edition 47(19): 3560-3564.

8. Yang SH, Lee KB, Kong B, Kim JH, Kim HS, et al. (2009) Biomimetic encapsulation of individual cells with silica. Angewandte Chemie International Edition 48(48): 9160-9163.

9. Yang SH, Ko EH, Jung YH, Choi IS (2015) Bioinspired functionalization of silica-encapsulated yeast cells. Angewandte Chemie International Edition 50(27): 6115-6118.

10. Park JH, Choi IS, Yang SH (2015) Peptide-catalyzed, bioinspired silicification for single-cell encapsulation in the imidazole-buffered system. Chemical Communications 51(25): 5523-5525.

11. Yang SH, Ko EH, Choi IS (2012) Cytocompatible encapsulation of individual chlorella cells within titanium dioxide shells by a designed catalytic peptide. Langmuir 28(4): 2151-2155.

12. Yang SH, Kang SM, Lee KB, Chung TD, Lee H, et al. (2011) Mussel-inspired encapsulation andfunctionalization of individual yeast cells. Journal of the American Chemical Society 113(9): 2795-2797.

13. Park JH, Kim K, Lee J, Choi JY, Hong D, et al. (2014) Cytoprotective and degradable metal-polyphenol nanoshell for single-Cell encapsulation. Angewandte Chemie International Edition 53(46): 12420-12425.

14. Mansouri S, Merhi Y, Winnik FM, Tabrizian M (2011) Investigation of layer-by-layer assembly of polyelectrolytes on fully functional human red blood cells in suspension for attenuated immune response. Biomacromolecules 12(3): 585-592.

15. Matsusaki M, Kadowaki K, Nakahara Y, Akashi M (2007) Fabrication of cellular multilayers with nanometer-sized extracellular matrix films. Angewandte Chemie International Edition 46(26) 4689-4692.

16. Veerabadran NG, Goli PL, Stewart-Clar SS, Lvov YM, Mills DK (2007) Nanoencapsulation of stem cells within polyelectrolyte multilayer shells. Macromolecular Bioscience 7(7): 877-882.

17. Germain M, Balaguer P, Nicolas JC, Lopez F, Esteve JP, et al. (2006) Protection of mammalian cell used in biosensors by coating with a polyelectrolyte shell. Biosensors and Bioelectronics 21(8): 1566-1573.

18. Swiston J, Cheng C, Um SH, Irvine DJ, Cohen RE, et al. (2008) Surface functionalization of living cells with multilayer patches. Nano Letters 8(12): 4446-4453.

19. Wilson JT, Krishnamurthy VR, Cui W, Qu Z, Chaikof EL (2009) Noncovalent cell surface engineering with cationic graft copolymers. Journal of the American Chemical Society 131(51): 18228-18229.

20. Wilson JT, Cui W, Kozlovskaya V, Kharlampieva E, Pan D, et al. (2011) Cell surface engineering with polyelectrolyte multilayer thin films. Journal of the American Chemical Society 133(18): 7054-7064.

21. Nishiguchi A, Yoshida H, Matsusaki M, Akashi M (2011) Rapid construction of three-dimensional multilayered tissues with endothelial tube networks by the cell-accumulation technique. Advanced Materials 23(31): 3506-3510.

22. Asano Y, Shimoda H, Okano D, Matsusaki M, Akashi M (2017) Transplantation of three-dimensional artificial human vascular tissues fabricated using an extracellular matrix nanofilm-based cellaccumulation technique. Journal of Tissue Engineering and Regenerative Medicine 11(4): 1303-1307.

23. Lee J, Choi J, Park JH, Kim MH, Hong D, et al. (2014) Cytoprotective silica coating of individual mammalian cells through bioinspired silicification. Angewandte Chemie International Edition 53(31): 8056-8059. 
24. Youn W, Ko EH, Kim MH, Park M, Hong D, et al. (2017) Cytoprotective encapsulation of individual Jurkat $\mathrm{T}$ cells within durable $\mathrm{TiO}_{2}$ shells for T-cell therapy. Angewandte Chemie International Edition 56(36) 10702-10706.

25. Lee J, Cho H, Choi J, Hong D, Kim D, et al. (2015) Chemical sporulation and germination: cytoprotective banocoating of individual mammalian cells with a degradable tannic acid-FelII complex. Nanoscale 7(45): 18918-18922.

26. Wang X, Sun C, Li P, Wu T, Zhou H, et al. (2016) Vaccine engineering with dual-functional mineral shell: a promising strategy to overcome preexisting immunity. Advanced Materials 28(4): 694-700.

\section{(c) (9) \\ This work is licensed under Creative Commons Attribution 4.0 License}

Submission Link: https://biomedres.us/submit-manuscript.php
27. Wang G, Wang HJ, Zhou H, Nian QC, Song Z, et al. (2015) Hydrated silica exterior produced by biomimetic silicification confers viral vaccine heatresistance. ACS Nano 9(1): 799-808.

28. Zhou H, Wang G, Li XF, Li Y, Zhu SY, et al. (2016) Alumina-encapsulated vaccine formulation with improved thermo stability and immunogenicity. Chemical Communications 52(38): 6447-6450.

\begin{tabular}{ll} 
BIOMEDICAL & Assets of Publishing with us \\
RESEARCHES & - Global archiving of articles \\
\hline ISS: $2574-1241$ & Immediate, unrestricted online access \\
\hline
\end{tabular}

